\title{
Evolución del mercado de seguros en Ecuador
}

\author{
Fecha de recepción: 2020-08-03 • Fecha de aceptación:2020-09-01 • Fecha de publicación: 2020-10-10
}

\author{
Alexander Alfonso Eras Piedra' ${ }^{1}$ \\ Corporación Nacional de Telecomunicaciones CNT, Ecuador \\ alexander.eras@cnt.gob.ec \\ https://orcid.org/0000-0002-5875-6885 \\ Ángel Gustavo Redrobán Chamorro² \\ Personalbrok Agencia de Seguros Cía. Ltda, Ecuador \\ angel.redroban@personalbrok.com \\ https://orcid.org/0000-0002-8913-3832
}

\section{Resumen}

Las compañías de seguros, como titulares de un importante capital económico, y por la importancia que tienen en el mercado ecuatoriano, se encuentran bajo la regulación de la Superintendencia de Compañías, Valores y Seguros, razón por la cual el objetivo del presente caso de estudio fue analizar la evolución del mercado de seguros en el Ecuador. Se utilizó la investigación descriptiva, basada en referencia bibliográfica documental que permitió establecer el crecimiento del mercado de seguros del $3.6 \%$ con respecto al año anterior, principalmente por el ramo de seguros Vida que en el año 2018 creció el $11.6 \%$ más, con respecto al 2017. A su vez, se evidenció que el segmento de seguros de vehículo ocupa el primer lugar en el mercado, con un $24 \%$. Es importante resaltar que el seguro privado dinamiza la economía, genera rentabilidad a sus propietarios y se ha convertido en indispensable para los consumidores, ante riesgos fortuitos.

\section{PALABRAS CLAVE: seguros, vehículos, riesgos, mercado de seguros.}

\section{Abstract}


Insurance companies, as holders of an important economic capital and due to the importance they have in the Ecuadorian market, are under the regulation of the Superintendency of Companies, Securities and Insurance, which is why the objective of this case study was analyze the evolution of the insurance market in Ecuador, for which a descriptive research based on documentary bibliographic references was used, which allowed establishing the growth of the insurance market of $3.6 \%$ with respect to the previous year, mainly due to the Life insurance branch that in the year 2018 grew $11.6 \%$ more compared to 2017. The vehicle insurance segment occupies the first place in the market, with $24 \%$. It is important to note that the private insurance boosts the economy, generates profitability for its owners and has become essential for consumers in the face of unforeseen risks. 


\section{Introducción}

Las compañías aseguradoras, como poseedoras de un importante capital económico y debido a la importancia que tienen dentro del mercado ecuatoriano, se encuentran bajo la regulación de la Superintendencia de Compañías y Seguros, qué como órgano de control, establece la normativa y los lineamientos para un correcto funcionamiento.

Para los ecuatorianos, la importancia de adquirir un seguro que les permita protegerse ante cualquier eventualidad que pueda afectarlos, es cada vez mayor, por lo que las compañías aseguradoras, en su búsqueda de captar clientes, bajo la característica del contrato de seguros como un contrato de adhesión, han iniciado la comercialización de sus productos de forma masiva a través de otras compañías o entidades ajenas al sector, aprovechando su cartera de clientes y los medios que estas poseen para ofrecer y distribuir sus productos.

El seguro se configura como la acción en virtud del cual, el asegurado (entiéndase por este como la persona, bien u objeto beneficiario de un seguro) se hace acreedor mediante el pago de una remuneración (prima) de una prestación, que habrá de satisfacer la otra parte llamado asegurador para prevenir o cuando se produzca un siniestro (Apolinario, R., \& Briones, V, 2015).

Actualmente ocurren accidentes a diario, que puede ser, por ejemplo, un incendio que perjudique a la sociedad, o que ocasione la muerte, por lo que adquirir un seguro se convierte en fundamental. Este no solo es de beneficio único, sino también puede ser usado por familiares, o a nivel de empresas, para que en el caso que ocurra algún siniestro, su patrimonio no se vea afectado en su totalidad y la familia no quede desamparada.

Bajo estas premisas, el objetivo de la presente investigación es analizar la percepción que se tiene sobre el mercado de los seguros, cómo este ha ido evolucionando con el pasar de los tiempos y la importancia que tiene en la economía ecuatoriana.

Inicialmente, es importante hacer una revisión bibliográfica de los significados que conllevan al tema propuesto.

Un seguro es un valor económico y comercial y a la vez un instrumento que permite a todas las personas, avizorar y garantizar la situación económica, ante hechos futuros, susceptibles de ocurrencia y de los que la persona que opta por él ni nadie puede tener certeza (Resico, 2010).

En esta parte cabe mencionar a los Brokers, también llamados corredores de seguros, agentes de seguros 0 asesores productores de seguros. Estas son las personas que desempeñan el papel de intermediarios entre el cliente y la aseguradora. Otorgan un servicio personalizado con el fin de facilitar los distintos procedimientos al cliente (Lucio, 2019).

Las empresas de seguros son solidariamente responsables por los actos ejecutados por los agentes de seguro (personas naturales) y las agencias asesoras productoras de seguros (personas jurídicas), de acuerdo a los contratos suscritos entre las partes. 
En cuento a la seguridad social, Resico (2010) explica que esta comprende la garantía de mejorar la capacidad de trabajo como base de un sustento seguro y responsable y lograr un adecuado nivel de seguridad social que permita a las personas obtener ingresos y un sistema de redistribución interpersonal e intertemporal de los ingresos en caso de necesidad justificada, la protección y recuperación de la salud. A su vez, garantizar la cantidad y la calidad de la educación, y compensar las diferencias en el nivel de ingresos y de cargas mediante una política redistributiva.

Para Delgado (2012), el riesgo es algo a lo que todas las personas, animales y cosas están expuestas pero cuyo acaecimiento puede suceder o no, es decir se trata de algo que es contingente $\mathrm{o}$ incierto.

Un primer hecho que debemos contemplar para obtener una imagen más adecuada del funcionamiento de una economía nacional es que las familias generalmente no consumen todo lo recibido en forma de ingresos, sino que una parte de ellos se puede destinar al ahorro (Resico, 2010).

"Una sociedad necesita desarrollar e implementar un orden social que corresponda a sus necesidades no económicas como la justicia social, la seguridad social y la paz social" (Resico, 2010, p.144). "Para lograr un "bienestar para todos", los actores de la economía, los empresarios, los consumidores, el gobierno, los sindicatos y las instituciones autónomas socioeconómicas, tienen que hacer el máximo esfuerzo para que todos los miembros de la sociedad puedan vivir en condiciones aceptables" (Cázares, 2008).

Para el autor Contreras (2008), en cuanto a la economía social del mercado de seguros y su incidencia en la economía, este "permite a las personas, empresas y otras organizaciones transferir sus riesgos, otorgándoles protección en caso de eventos que les provoquen daños patrimoniales o en su integridad física, generando alternativas de ahorro y previsión para las familias, favoreciendo el desarrollo de la actividad económica en un ambiente de confianza y fomentando el desarrollo del mercado de capitales a través de la inversión de los recursos que administra, lo que finalmente se traduce en mayor riqueza y bienestar para un país".

\section{Metodología}

El presente caso de estudio utilizó la investigación descriptiva. Según el autor Frank Morales (2012) este tipo de investigación busca definir claramente un objeto, el cual puede ser un mercado, una industria, una competencia, puntos fuertes o débiles de empresas, algún tipo de medio de publicidad o un problema simple de mercado.

De esta manera se analizaron fuentes bibliográfica-documental de acuerdo al segmento del mercado de los seguros del lado de la demanda, siendo estos los consumidores (asegurados) y a su vez de la oferta de los seguros de vehículos por parte del bróker que permitió establecer resultados en base a la información obtenida de la Superintendencia de Compañías, Valores y Seguros. 


\section{Resultados}

De acuerdo a la información recolectada sobre las aseguradoras del sector privado en la Superintendencia de Compañías, Valores y Seguros (SCVS), el volumen de primas netas emitidas del mercado asegurador ecuatoriano alcanzó los 1,689 millones de USD en el año 2018, lo cual representa un crecimiento del $3.6 \%$ con respecto al año anterior (Figura 1). Esto se debe al crecimiento que tuvieron ambos de sus ramos, Vida y No Vida, impulsado principalmente por el ramo de seguros Vida que por tercer año consecutivo ha venido creciendo, reportando 412.7 millones de USD en primas netas emitidas en el año 2018, es decir, un $11.6 \%$ más con respecto al 2017.
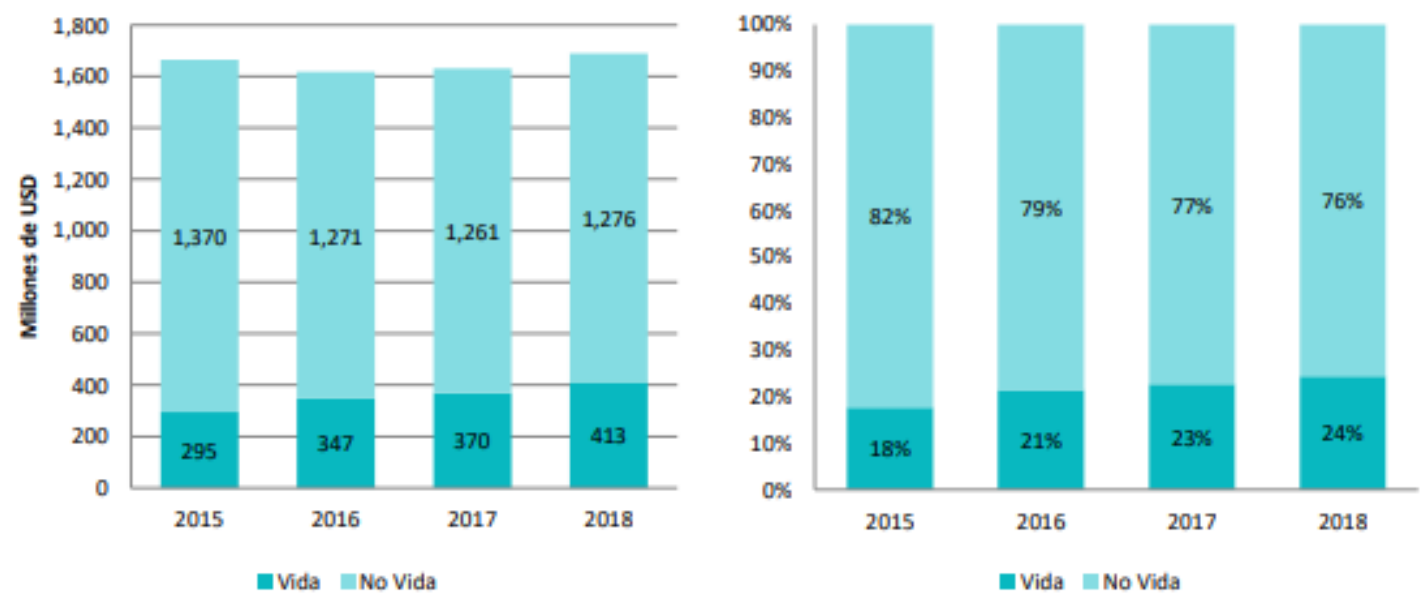

Figura 1. Crecimiento del mercado de seguros

Fuente: elaboración propia

El segmento de seguros de vehículos (Figura 2) continúa posicionándose como el seguro que acumula el mayor volumen del mercado asegurador en cuanto a la prima neta emitida con una cuota promedio del $24 \%$ durante el período 2015-2018. 


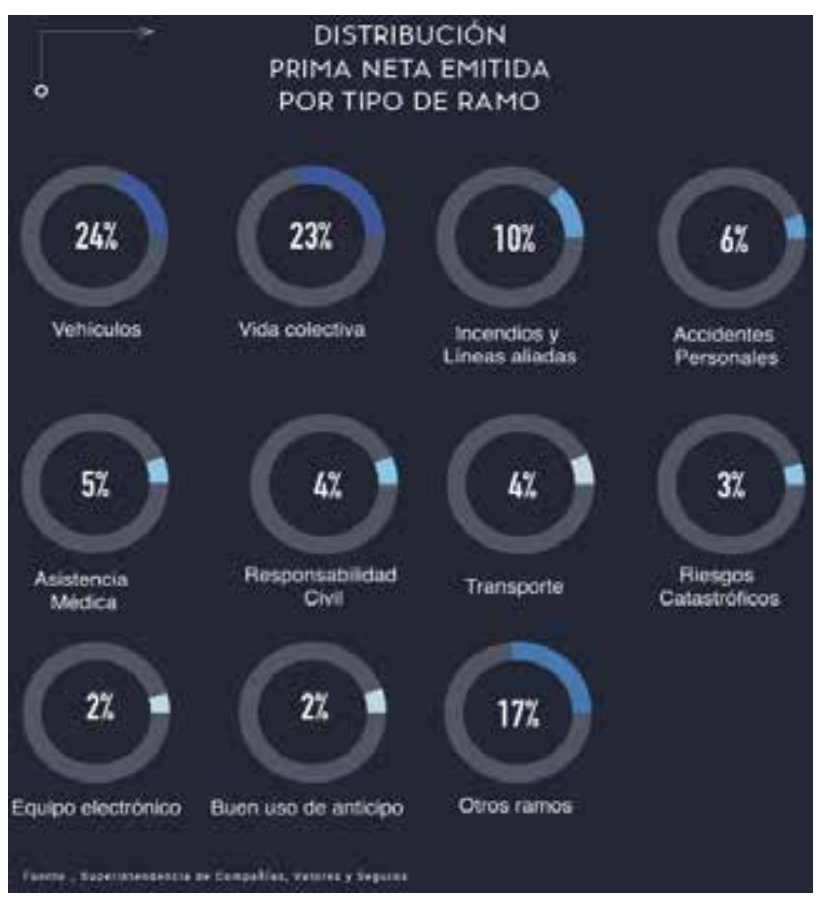

Figura 2. Posicionamiento de seguros de vehículos

Fuente: elaboración propia

A su vez, en los últimos cuatro años (Figura 3) su evolución en relación a la prima neta emitida ha sufrido variantes; en el período 2015 registró un monto de 438 millones de USD; mientras que en el año 2016 el nivel de la prima disminuyó al obtener 383 millones de USD representando un decrecimiento de 12.5\% (Superintendencia de Compañía, 2019).

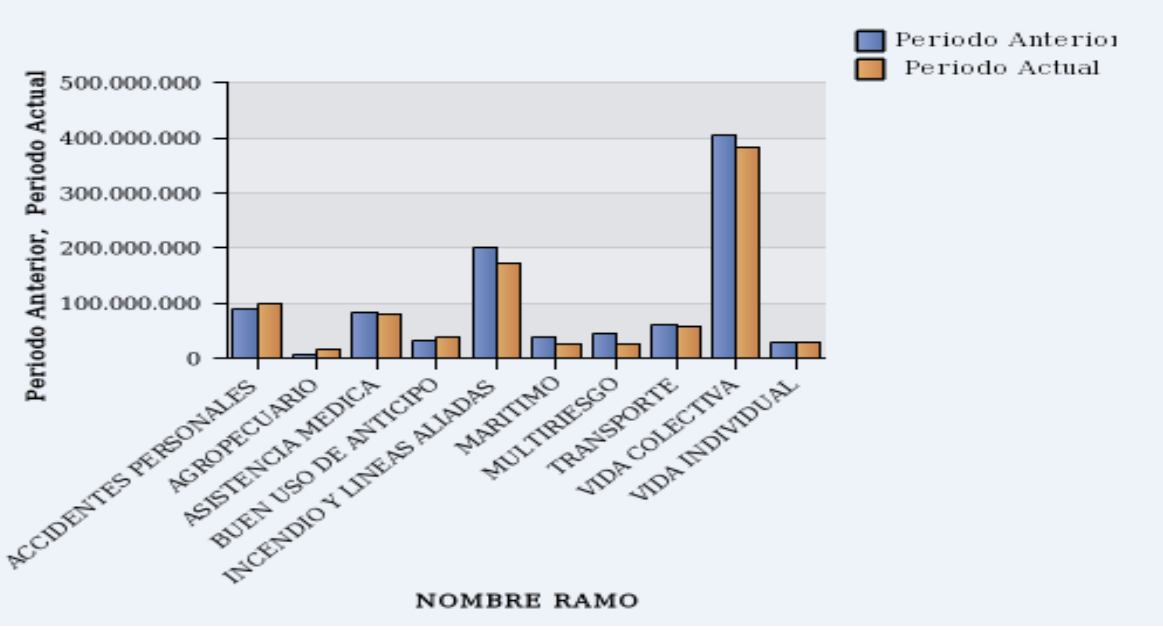

Figura 3. Evolución del seguro con relación a la prima neta

Fuente: elaboración propia 
En el período 2017 el volumen de primas disminuyó por segundo año consecutivo hasta llegar a los 379 millones de USD con un decrecimiento de 1.2\%; por otra parte, en el año 2018 la prima neta emitida incrementó en un 5.7\% de variación anual alcanzando los 385 millones de USD; sin embargo, este no alcanza al monto de primas netas emitidas a inicios del periodo analizado, ya que presenta una caída del 12\% respecto al año 2015.

Por otra parte, de acuerdo a la información registrada por el Instituto Nacional de Estadística y Censos - INEC (2019), en la provincia de pichincha se cuenta con un total de 540.827,00 vehículos registrados hasta el 2018 (Tabla 1).

Tabla 1.

Evolución del seguro con relación a la prima neta

\begin{tabular}{llll}
\hline $\begin{array}{l}\text { Provincia de } \\
\text { matriculación } \\
\text { vehicular }\end{array}$ & $\begin{array}{l}\text { Número de vehículos } \\
\text { matriculados }\end{array}$ & Población año 2018 & $\begin{array}{l}\text { Número de vehículos } \\
\text { matriculados por } \\
\text { cada 1000 habitantes }\end{array}$ \\
\hline Pichincha & 540.827 & 3.116 .111 & 174 \\
\hline
\end{tabular}

El dato de la

población se obtiene

de las proyecciones

poblacionales

publicadas por el INEC.

Fuente: elaboración propia

En la Tabla 2 se puede evidenciar que durante el año 2018 se presentaron un total de 7.599 siniestros de transito de la provincia de pichincha, según datos de la Agencia Nacional de Tránsito (ANT).

Tabla 2.

Siniestros de tránsito en Pichincha

\begin{tabular}{|c|c|c|c|c|c|c|c|c|c|c|c|c|c|}
\hline \multirow[t]{2}{*}{ Provincia } & \multirow[t]{2}{*}{ Total } & \multicolumn{12}{|c|}{ Meses } \\
\hline & & 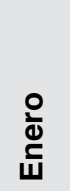 & 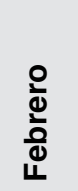 & $\frac{\stackrel{8}{\mathbb{N}}}{\stackrel{N}{\frac{N}{2}}}$ & $\begin{array}{l}\bar{\Sigma} \\
\frac{2}{\alpha}\end{array}$ & 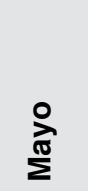 & $\stackrel{\circ}{\stackrel{0}{5}}$ & $\stackrel{\circ}{3}$ & $\begin{array}{l}\text { 劳 } \\
\text { 总 }\end{array}$ & 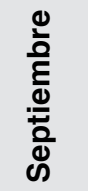 & 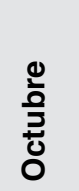 & $\begin{array}{l}\frac{0}{0} \\
\frac{0}{E} \\
\frac{\omega}{2} \\
\frac{0}{2}\end{array}$ & 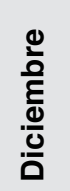 \\
\hline ichincha & 75 & 648 & 629 & 698 & 696 & 631 & 656 & 628 & 613 & 668 & 621 & 539 & 572 \\
\hline
\end{tabular}

Fuente: elaboración propia

En la Tabla 3 se detalla que en el mismo año se presentaron un total de 4.878 víctimas en accidentes de tránsitos de los cuales 357 personas fallecieron y 4.521 personas resultaron lesionados en la provincia de pichincha. 
Tabla 3.

Víctimas de Siniestros de tránsito en Pichincha

\begin{tabular}{llll}
\hline Provincia & Total de víctimas & Fallecidos & Lesionados \\
\hline Pichincha & 4.878 & 357 & 4.521 \\
\hline
\end{tabular}

Fuente: elaboración propia

\section{Factores que afectan al desarrollo de mercado de seguros en Quito}

El desconocimiento del mercado es uno de los factores más importantes que impide que el sector de los seguros no se desarrolle de una mejor manera, debido que las aseguradoras al momento de entrar al mercado deben hacer una investigación de mercado, donde evaluaran los competidores que existen, los posibles o futuros consumidores de seguros, diagnosticar si el desarrollo del negocio asegurador es dinámico conforme va evolucionado y desarrollando la sociedad, ya que este factor está íntimamente relacionado con los consumidores, porque si las compañías de seguros no tienen un amplio conocimiento del mercado al cual están promocionando sus coberturas, mucho menos los tendrán los futuros consumidores, generando así que este sector no sea tan amplio y los consumidores desconozcan de los atributos y beneficios que este pueda brindarle.

Muchas familias, y la sociedad en general, no conoce a lo que se expone en el vivir diario, esto representa una amenaza debido a que el peor riesgo por el cual las personas atravesarían es aquel que se desconoce, ya que su impacto puede ser muy fuerte sin poder reponerse, las personas carecen de conciencia de riesgo y de las posibles consecuencias que pueda suscitarse ante un siniestro.

A su vez, el marketing digital es la última revolución del mundo empresarial y se ha convertido en una herramienta clave potenciar de manera eficiente cualquier negocio, marca o servicio (Pérez, 2020). Por medio de este se hace posible que los consumidores conozcan el producto o servicio que la compañía ofrece en el mercado.

Otra limitante es que hay que estar consciente que el cliente evalúa el profesionalismo que ofrece la compañía aseguradora mediante el primer contacto, por lo que los agentes de seguros deben estar debidamente capacitados día a día, ya que el mercado no es dinámico, siempre existen nuevas exigencias por parte de los clientes, el agente de seguros debe de tener un amplio conocimiento de los diferentes seguros que ofrecen las distintas compañías de seguros (Cevallos, 2014).

\section{Conclusiones}

De la incidencia que existe entre el seguro y la economía, este es de gran aporte para las variables macroeconómicas, tanto para la balanza de pagos en el sector externo, como herramienta canalizadora de ahorros por parte de los seguros de vida, los cuales juegan un papel fundamental en la sociedad. 
El mercado asegurador privado ecuatoriano cuenta con 25 empresas aseguradoras de vehículos, lo cual representa proporcionalmente la tercera parte respecto al total de seguros, con una siniestralidad promedio registrada históricamente $(2015$ - 2018) en el seguro vehicular ecuatoriano. Aclarando que la ciudad de Quito carece de coberturas totales para todo el distrito metropolitano.

Las inversiones que realizan las compañías de seguros sirven como fomento para el crecimiento económico, ya que el dinero captado por las primas de seguros debe de ser respaldado por lo cual las aseguradoras realizan inversiones, donde el sector financiero es el de mayor concentración, ya sea en compras de acciones, bonos, títulos, lo cual a su vez genera que las compañías sean más rentables, que el dinero circule en la economía y así ayuda a varios sectores de la economía.

Las compañías de seguros en los últimos años han designado fuertes cantidades de dinero a las reservas técnicas, las cuales sirven para cubrir los siniestros que se presenten en algún contrato de seguros, el cual sirve de respaldo y está directamente relacionado con las inversiones que realizan las aseguradoras, donde la mayor compañía de seguros de participación en el mercado. 


\section{Referencias}

Agencia Nacional de Tránsito. (2018). Estadísticas de siniestros de tránsito. https://ant.gob.ec/index.php/estadisticas

Apolinario, R., \& Briones, V. (2015). Seguro y Logística Internacional. Guayaquil-Ecuador: Palibrio.

Cázares, R. (2008). El enfoque por competencias en eduación. Revista electrónica de desarrollo de competencias, $10-20$.

Cevallos, A. (2014). Análisis de la evolución del mercado de seguros de Guayaquil y su incidencia en la economía periodo 2006-2012. http://repositorio.ucsg.edu.ec/bitstream/3317/1446/1/T-UCSG-PRE-ECO-CECO-5.pdf

Contreras, O. (2008). Temas relevantes del derecho de seguros contemporáneo. Madrid.: Páginas 601. https:// app.mapfre.com/ccm/content/documentos/fundacion/cs-seguro/libros/Temas_relevantes_del_Derecho_ de_Seguros_contemporaneo_CILA-129.pdf

Delgado, H. M. (2012). Seguros y finanzas para la familia. Ecoe Ediciones.

Instituto Nacional de Estadística y Censos. (2019). Anuario de Estadísticas de Transporte 2018. https://www. ecuadorencifras.gob.ec/documentos/web-inec/Estadisticas_Economicas/Estadistica\%20de\%20Transporte/2018/2018_ANET_PPT.pdf

Lucio, M. (2019). Diferencias entre Broker y Aseguradora. Seguros123.com. https://ecuador.seguros123.com/ broker-vs-aseguradora/

Morales, F. (2012). Conozca 3 tipos de investigación: Descriptiva, Exploratoria y Explicativa. Recuperado el, 11.

Pérez, S. (2020). Marketing Digital: la herramienta indispensable para que tu negocio triunfe. The butterfly webs. https://thebutterflywebs.com/blog-marketing-digital/marketing-digital-la-herramienta-indispensable-negocio-triunfe/

Resico, M. (2010). Introducción a la Economía Social del Mercado. Buenos Aires.: Solugraf . https://www.kas. de/c/document_library/get_file?uuid=22412104-f255-886e-178f-8b32bf5bce06\&groupld=252038

Superintendencia de Compañía, V. y. (2019). Reporteria Superintendencia de Compañias. https://reporteria. supercias.gob.ec/portal/cgi-bin/cognos.cgi\# 